\title{
КАРДИОЛОГИЯ GARDIOLOGY
}

УДК 616.12-008.331.1(533/534)

DOI: $10.18413 / 2687-0940-2020-43-3-373-382$

\section{Факторы риска больных артериальной гипертонией, жителей Республики Йемен}

\author{
В.И. Вишневский ${ }^{1}$, А.А.А. Исмаил ${ }^{1}$, М.А. Аль-Гунаид ${ }^{2}$, Х.А.Ф. Саид ${ }^{3}$ \\ ${ }^{1}$ Орловский государственный университет имени И.С. Тургенева, \\ Россия, 302026, г. Орел, ул. Комсомольская, 95 \\ ${ }^{2}$ Волгоградский государственный технический университет, \\ Россия, 400005, г. Волгоград, пр. Ленина, 28 \\ ${ }^{3}$ Национальный исследовательский Мордовский государственный университет \\ имени Н. П. Огарёва, \\ Россия, 430005, Республика Мордовия, г. Саранск, ул. Большевистская, 68 \\ E-mail: badani20@gmail.com
}

\begin{abstract}
Аннотация. Изучены наиболее значимые факторы риска (ФР) артериальной гипертонии (АГ) среди жителей Республики Йемен. Проведен многофакторный анализ для ассоциации различных переменных ФР у 101 пациента АГ, жителей Республики Йемен, средний возраст - 54,3 \pm 9,3 года. Наиболее часто среди ФР АГ встречались низкий доход - у 66,3\%, употребление ката - у 64,3 \%, избыточная масса тела - у 42,5 \%; отягощенная наследственность - у $35 \%$; наличие нарушения углеводного обмена - у $20 \%$; курение - у 19,8 \% пациентов. Среди употреблявших кат средний уровень АД составил 163 и 95 мм рт. ст., причем более высокий уровень АД наблюдался у лиц мужского пола. Показана сильная прямая связь вредных привычек (употребление ката), менее выраженная связь социально-экономических условий (проживание в районах, где происходят боевые действия, перемещенные лица, неудовлетворительные жилищные условия, низкий материальный доход) и степенью повышения АД. Не выявлено корреляционных связей с полом, возрастом больных АГ. Полученные данные позволят актуализировать немедикаментозное лечение АГ у жителей Республики Йемен.
\end{abstract}

Ключевые слова: артериальная гипертония, факторы риска, многофакторный анализ, жители республики Йемен.

Для цитирования: Вишневский В.И., Исмаил А.А.А., Аль-Гунаид М.А., Саид Х.А.Ф. 2020. Факторы риска больных артериальной гипертонией, жителей Республики Йемен, 43 (3): 373-382. DOI: $10.18413 / 2687-0940-2020-43-3-373-382$

\section{Risk factors for patients with arterial hypertension, residents of the Republic of Yemen}

\author{
Valery I. Vishnevsky ${ }^{1}$, Aref Ahmed Abdullah Esmail ${ }^{1}$, Mohammed Amin Al-Gunaid ${ }^{2}$, \\ Hashem Anwar Fayd Saeed ${ }^{3}$ \\ ${ }^{1}$ Orel State University named after I.S. Turgenev, 95 Komsomolskaya St., Oryol, 302026, Russia \\ ${ }^{2}$ Volgograd State Technical University, 28 Lenin Ave., Volgograd, 400005, Russia \\ ${ }^{3}$ Ogarev Mordovia State University, 68 Bolshevik St., Saransk, 430005, Republic of Mordovia, Russia \\ E-mail: badani20@gmail.com
}

Abstract. The most significant risk factors (FR) of arterial hypertension (AH) among residents of the Republic of Yemen were studied. A multivariate analysis was performed for the Association of various FR 
variables in $101 \mathrm{AH}$ patients, residents of the Republic of Yemen, with an average age of $54.3 \pm 9.3$ years. Low income in $66.3 \%$, chewing khat (qat) in $64.3 \%$, excess body weight in $42.5 \%$, burdened heredity in $35 \%$, presence of carbohydrate metabolism disorders in $20 \%$, Smoking in $19.8 \%$ of patients were the most common among AH patients. Among khat users, the average blood PRESSURE was 163 and $95 \mathrm{mmHg}$. however, a higher level of blood PRESSURE was observed in males. There is a strong direct relationship between bad habits (chewing khat), less pronounced relationship between socio-economic conditions (living in areas where there is fighting, displaced persons, poor housing conditions, low material income) and the degree of increase in blood PRESSURE. There were no correlations with gender and age of patients with hypertension. The obtained data will help to update non-drug treatment of hypertension in the residents of the Republic of Yemen.

Keywords: arterial hypertension, risk factors, many factors analysis, residents of Yemen.

For citation: Vishnevsky V.I., Esmail A.A.A., Al-Gunaid M.A., Saeed H.A.F. 2020. Risk factors for patients with arterial hypertension, residents of the Republic of Yemen. Challenges in Modern Medicine, 43 (3): 373-382 (in Russian). DOI: 10.18413/2687-0940-2020-43-3-373-382

\section{Введение}

Артериальная гипертония (АГ) - одно из самых частых заболеваний сердечнососудистой системы (ССС). АГ страдают 20-30 \% взрослого населения планеты; с возрастом распространённость болезни увеличивается и достигает 50-65 \% у лиц старше 65 лет [Климов и др., 2018].

АГ способствует поражению органов-мишеней и развитию целого ряда осложнений ССС (инфаркт миокарда, мозговой инсульт, сердечная недостаточность и др.), приводящих к смертности до 7,6 млн пациентов в год во всем мире [Чазова, Жернакова, 2019; World Health Organization, 2009; Lawes CM et. al., 2008].

К 2025 году ожидается значительное увеличение числа пациентов АГ (на 15-20 \%) наряду с ростом распространенности ожирения, малоподвижного образа жизни и других факторов риска (ФР) на фоне старения населения земного шара в целом [Willams, Mancia, 2018].

В странах с высоким уровнем дохода, с широкой доступностью современной лабораторно-инструментальной диагностики и возможностью лечения эффективными антигипертензивными препаратами (АГП) больных АГ отмечено значительное сокращение пациентов с неконтролируемым уровнем АД и снижение осложнений ССС [Европейские рекомендации, 2016].

В странах с низким уровнем дохода имеет место самая высокая распространенность неконтролируемого АД. Общая распространенность АГ в Африке в 2017 году составила 55,2 \% по сравнению с США [Arnaud D. Kaze et. al., 2017; Centers for Disease Control and Prevention, 2017]. По некоторым данным ВО3, 32-51 \% взрослого населения арабских стран страдают АГ [Who, Prevalence Of Hypertension Worldwide 2017].

Артериальная гипертония (АГ) имеет широкое распространение в странах арабского мира, заболеваемость АГ неуклонно растет (рис. 1).

Многие жители развивающихся стран, в том числе республики Йемен, с высоким уровнем АД не знают о своем заболевании, не имеют доступа к современному обследованию и лечению эффективными АГП [Kearney P. et. al., 2004; Chow CK et. al., 2013]. В ocнове лежат различные причины, к которым можно отнести психологические особенности пациентов, качество оказания и доступность медицинских услуг, а также уровень дохода населения, место проживания и многие другие [Baker R. et. al., 2010; Nieuwlaat R. et. al., 2013]. Своевременная диагностика, эффективное лечение АГ, контроль целевого уровня АД, протекция органов-мишеней является важным приоритетом практической кардиологии во всем мире. 


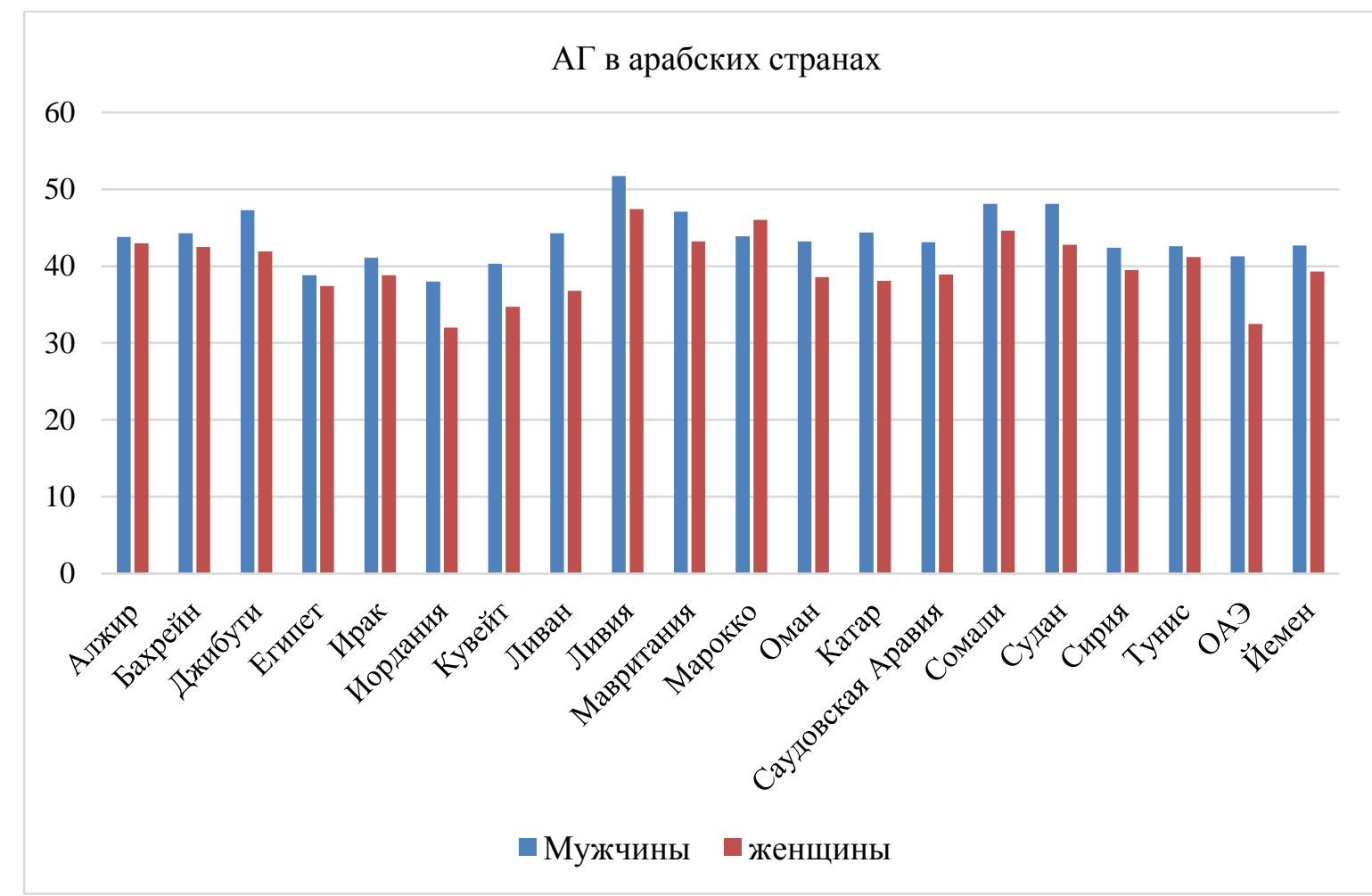

Рис. 1. Показатели частоты повышенного АД среди жителей старше 25 лет ряда арабских стран Fig. 1. Indicators of the frequency of high blood pressure among residents over 25 years of age in several Arab countries

Факторы риска (ФР) АГ в республике Йемен имеют свои особенности - употребление ката, проживание в районах, где происходят боевые действия, перемещенные лица, неудовлетворительные жилищные условия, низкий материальный доход, что повышает частоту депрессивных состояний, постоянного чувства тревоги и др. [Ahmed Hasan, 2014].

По некоторым оценкам ВОЗ, в Йемене кат употребляет до 90 \% мужчин, 25-50 \% женщин и даже 15-20\% детей и подростков. Для самих йеменцев жевание ката - это стиль жизни [WHO-2008]. Кат обладает симпатомиметическим и эйфориантным эффектами [Al-huthi M. et. al., 2016]. Основным компонентом ката является алкалоид катинон, точкой приложения которого является ССС. Катинон структурно и функционально похож на амфетамин [El-Menyar Ayman et. al., 2015]. При хроническом употреблении ката имеет место стойкое повышение АД за счет катинона, катина, эфедрина, которые способствуют сужению кровеносных сосудов, увеличению частоты сердечных сокращений, частоты дыхания, повышению температуры тела, расширению зрачка. Пик повышения АД и ЧСС наступает через 3 часа после начала употребления ката, и начинается возвращаться к исходному уровню через час после прекращения употребления ката [Ahmed Hasan, 2014].

Кроме того, необходимо учитывать некоторые местные особенности жителей арабских стран, например, соблюдение поста Рамадан. С одной стороны, Рамадан - это хороший повод навести порядок во всем: привести в соответствии с нормами духовную и физическую жизнь, изменить образ жизни, отказаться от вредных привычек. С другой нарушается привычный образ жизни [Mohtar Al-taher Hasan, 2005].

Цель работы: изучить особенности и частоту различных факторов риска у больных артериальной гипертонией, жителей республики Йемен.

\section{Задачи исследования:}

1. Изучить медико-социальные характеристики больных с АГ. 
2. Оценить роль ФР в развитии АГ у жителей республики Йемен. Йемен.

3. Провести многофакторный анализ ФР у больных АГ жителей республики

\section{Объекты и методы исследования}

\section{Объект исследования}

Исследование проводилось на базе отдела терапии городского медицинского учреждения - госпиталь Аль-Таура, г. Ибб, Республика Йемен.

Обследован 101 больной первичной АГ, средний возраст - 55,9 $\pm 2,1$ лет. Характеристика больных представлена в таблице 1.

Таблица 1

Table 1

Исходная характеристика больных

Initial characteristics of patients

\begin{tabular}{|c|c|c|c|}
\hline \multicolumn{2}{|c|}{ Показатели } & Мужчины & Женщины \\
\hline \multicolumn{2}{|l|}{ Количество } & $\mathrm{n}=45(44,5 \%)$ & $\mathrm{n}=56(55,4 \%)$ \\
\hline \multirow[t]{3}{*}{ Возраст } & Средний возраст & $55,5 \pm 2,1$ & $53,3 \pm 2,7$ \\
\hline & Моложе 55 лет & $20(44,4 \%)$ & $26(46,4 \%)$ \\
\hline & Старше 55 лет & $25(55,5 \%)$ & $30(53,5 \%)$ \\
\hline \multirow[t]{3}{*}{ Профессия } & Пенсионеры/ки & $22(19,8 \%)$ & $16(15,8 \%)$ \\
\hline & Домохозяйки & --- & $36(35,6 \%)$ \\
\hline & Имели государственную или частную работу & $23(11,8 \%)$ & $4(7,1 \%)$ \\
\hline \multicolumn{2}{|c|}{ Средний ИМТ (кг/м²) } & $\begin{array}{c}24,39 \pm 3,3 \\
\kappa \Gamma / \mathrm{M}^{2}\end{array}$ & $24,88 \pm 2,8$ кг $/ \mathrm{M}^{2}$ \\
\hline \multicolumn{2}{|c|}{ Отягощенная наследственность по АГ } & $16(35,5 \%)$ & $20(35,7 \%)$ \\
\hline \multicolumn{2}{|c|}{ Нарушение углеводного обмена (сахарный диабет) } & $11(24,4 \%)$ & $10(17,8 \%)$ \\
\hline \multicolumn{2}{|c|}{ Курение } & $17(37,7 \%)$ & $3(5,3 \%)$ \\
\hline \multicolumn{2}{|c|}{ Употребление ката } & $37(82,2 \%)$ & $28(50 \%)$ \\
\hline \multicolumn{2}{|c|}{ Проживание в территориях, где ведутся военные действия } & $8(17,7 \%)$ & $9(16,0 \%)$ \\
\hline \multicolumn{2}{|c|}{ Перемещённое лицо } & $14(31,1 \%)$ & $24(42,8 \%)$ \\
\hline \multirow{2}{*}{$\begin{array}{l}\text { Материальный } \\
\text { достаток }\end{array}$} & Низкий доход & $12(26,0 \%)$ & $17(30,3 \%)$ \\
\hline & Средний доход & $16(35,5 \%)$ & $22(39,2 \%)$ \\
\hline \multirow{3}{*}{$\begin{array}{l}\text { Условия } \\
\text { проживания }\end{array}$} & Плохие & $12(26,0 \%)$ & $17(30,3 \%)$ \\
\hline & Удовлетворительные & $16(35,5 \%)$ & $22(39,2 \%)$ \\
\hline & Нормальные & $17(37,7 \%)$ & $17(30,3 \%)$ \\
\hline \multicolumn{2}{|l|}{ Знали о АГ } & $5(4,9 \%)$ & $8(7,9 \%)$ \\
\hline
\end{tabular}

\section{Критерии исследования}

Критерии включения в исследование: первичная артериальная гипертония 1-2 степени, 1-2 стадии, не получающие антигипертензивную терапию (впервые диагностированная АГ), возраст пациентов 35-70 лет, СКФ более 60 мл/мин.

Критерии исключении: вторичная АГ, ишемическая болезнь сердца (стенокардия, инфаркт миокарда), ожирение выше 1 ст., тяжелая почечная и печеночная недостаточность, беременность, лактация.

\section{Сбор и обработка данных}

В ходе выполнения данной работы применена обычная анкета, в которую вносили все данные больных и был исследован ряд ФР АГ: пол, возраст, профессия, ИМТ, наследственная отягощенность по АГ, курение, употребление ката, социально-экономические условия (проживание на территориях, где ведутся военные действия; низкий доход и др.), нарушение углеводного обмена. 


\section{Статистический анализ}

В данной работе математическая обработка полученных данных исполнена с поддержкой электронных таблиц Microsoft Excel 7.0. Вычисление средних арифметических исследуемых показателей и их стандартных отклонений, корреляционных взаимосвязей (r) между АГ и ее ФР, многофакторный анализ факторов риска АГ проводились с использованием MSExcel, Deductor, SPSS Statistics и языка программирования Python. Распределенные статистические показатели были выражены как среднее \pm стандартное отклонение (SD). Для сравнения между двумя группами был принят t-критерий, для сравнения между несколькими группами был использован односторонний дисперсионный анализ, а для парного сравнения между несколькими группами - q-критерий. $\mathrm{P}<0,05$ считалось статистически значимым.

\section{Результаты исследования и их обсуждение}

Как следует из таблицы 1 , наиболее значимым распространенным ФР среди пациентов с АГ среди городских жителей Республики Йемен было употребление ката - 64,3 \%; средний уровень АД для данной категории больных составил 163 и 95 мм рт. ст. Нетрудоспособный возраст отмечен у 54,4 \%, отягощенная наследственность - у 35,8 \%, низкий доход - у 66,3 \% больных. Практически все факторы риска чаще встречались у лиц мужского пола. Не было выявлено гендерных отличий в распространенности немодифицируемого ФР - отягощенной наследственности по АГ. Отмечена низкая информативность больных о АГ в Республики Йемен (менее $10 \%$ ).

Течение АГ характеризовалось нестабильными цифрами АД у пациентов, употребляющих кат, и это в большей степени было характерно для мужчин. Не было выявлено достоверной зависимости курения табака и исходных цифр АД (рис. 2).

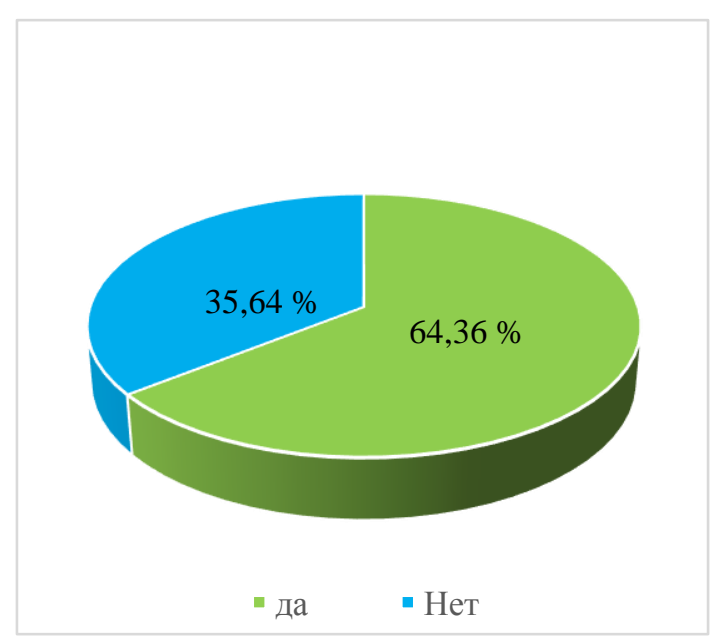

а) Употребление ката

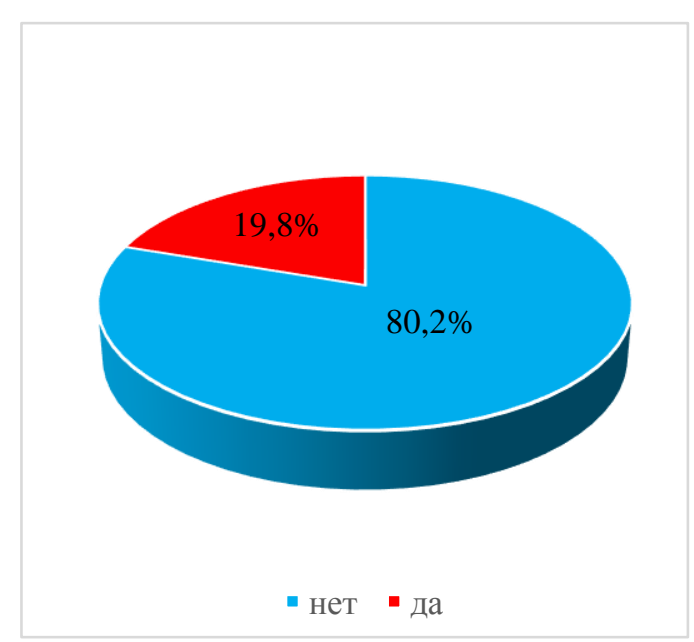

б) Курение

Рис. 2. Сравнительные данные по вредным привычкам

Fig. 2. Comparative data on bad habit

Полученные данные показали тесную взаимосвязь АГ с социальноэкономическими условиями: последствия войны - 54,45 \% больных (проживание на территориях, где ведутся военные действия, перемешенные лица); низкий доход - 66,33\% больных (плохие, удовлетворительные условия проживания) и др. (рис. 3). 


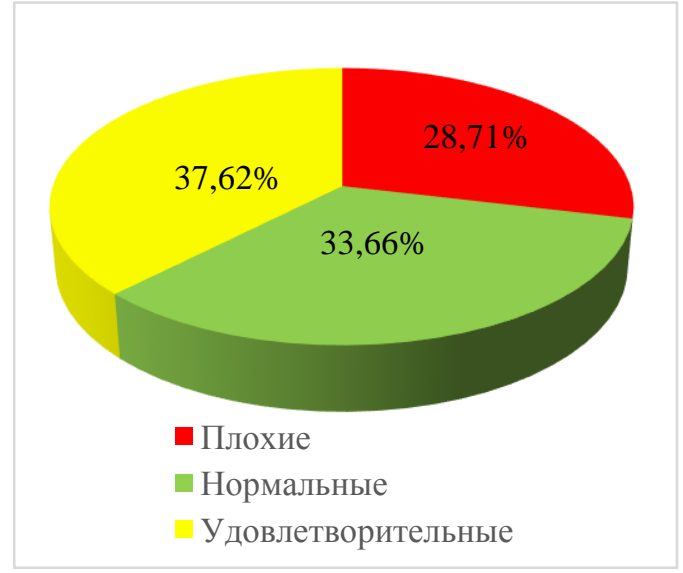

а) Условия проживания

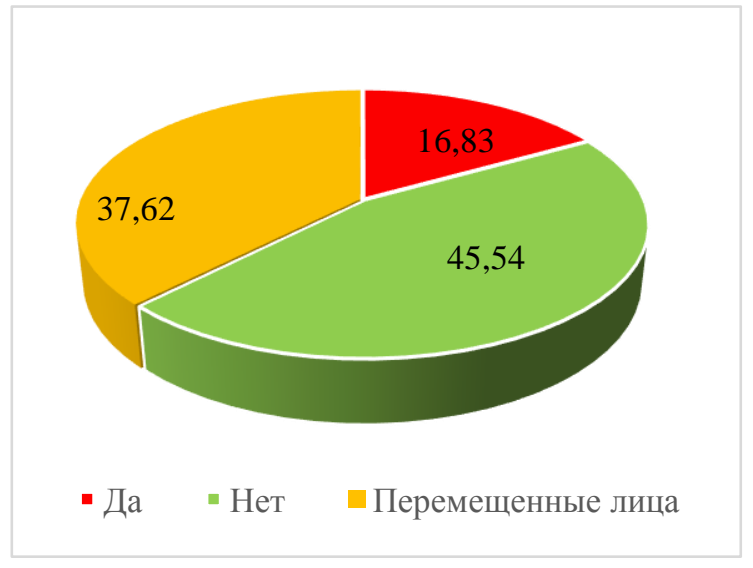

б) Проживание на территориях, где ведутся военные действия и перемешенные лица

Рис. 3. Сравнительные данные по социально-экономическим показателям

Fig. 3. Comparative data on socio-economic indicators

Проведен многофакторный анализ факторов риска больных АГ. Главными целями факторного анализа являются: 1) сокращение числа переменных (факторы риска) и 2) определение структуры взаимосвязей между переменными, т. е. классификация переменных (см. таб. 2).

Таблица 2

Table 2

Многофакторный анализ факторов риска больных АГ

Many factors analysis of risk factors for patients with hypertension

\begin{tabular}{|l|c|c|c|c|}
\hline \multicolumn{2}{|c|}{ Переменные факторы } & \multicolumn{3}{c|}{ Компоненты } \\
\cline { 2 - 5 } & 1 & 2 & 3 & 4 \\
\hline Пол & $-0,786$ & & & \\
\hline Курение & $0,769^{*}$ & & & \\
\hline Употребление ката & 0,685 & & & \\
\hline Возраст & & $-0,855$ & & \\
\hline Отягощенная наследственность & & 0,73 & & \\
\hline Профессия & & 0,664 & & $0,361^{*}$ \\
\hline Низкий доход & & & $-0,851$ & \\
\hline Проживание на территориях, где ведутся военные действия & & & 0,819 & \\
\hline Нарушение углеводного обмена & & & & 0,897 \\
\hline
\end{tabular}

Примечание: * $-\mathrm{p}<0,05$.

В таблице 2 показана матрица ФР после вращения, которая включает в себя 4 компонента. Данные многофакторного анализа показали, что отдельные факторы риска АГ не всегда являются единственными и независимыми предикторами высокого АД. Если рассматривать все факторы риска вместе, то новые факторы риска (4 компоненты), которые объединяют несколько взаимосвязанных между собой переменных факторов играют важную роль в развитии АГ у жителей Республики Йемен.

1-й компонент имеет прочные связи с 3 из 10 переменных факторов. Это значит, что пол и вредные привычки (курение и употребление ката) взаимосвязаны между собой и играли большую роль в возникновении АГ в 1 компоненте. 
2-й компонент имеет прочные связи с 3 из 10 переменных факторов. Возраст, отягощенная наследственность и профессия взаимосвязаны между собой и также считаются главными факторами риска АГ во 2 компоненте.

3-й компонент имеет сильные отношения с 2 из 10 переменных факторов. Социальноэкономические влияние (низкий доход; проживание на территориях, где ведутся военные действия) взаимосвязаны друг с другом и также сильно влияют на АГ в 3 компоненте.

4-й компонент имеет сильные отношения с 1 из 10 переменных факторов. Нарушение углеводного обмена в 4 компоненте участвовало в возникновении АГ у жителей Йемена.

Таблица 3

Table 3

Корреляционный анализ показателей АГ и ее ФР

Correlation analysis of arterial hypertension and its risk factors

\begin{tabular}{|l|c|}
\hline \multicolumn{1}{|c|}{ Факторы риска } & Корреляционная взаимосвязь (r) \\
\hline Пол & $0.077^{*}$ \\
\hline Возраст & $-0,137$ \\
\hline Профессия & $-0,018$ \\
\hline ИМТ & 0,224 \\
\hline Употребление ката & $0,934^{*}$ \\
\hline Курение & 0,105 \\
\hline Социально-экономические условия & $0,428^{*}$ \\
\hline Наследственная отягощенность & $-0,089$ \\
\hline Нарушение углеводного обмена & 0,053 \\
\hline
\end{tabular}

Примечание: $*-\mathrm{p}<0,05$.

По данным в таб. 3. выявлена положительная корреляционная связь вредных привычек (употребление ката) $(\mathrm{r}=0,93425)$, социально-экономических условий $(\mathrm{r}=0,42889)$ и степенью повышения АД.

\section{Заключение}

Наше исследование было посвящено корреляционному и многофакторному анализу ФР у больных АГ, коренных жителей Республики Йемена. Впервые проведен многофакторный анализ ФР у этой категории пациентов. Показана сильная прямая связь вредных привычек (употребление ката) $-\mathrm{r}=0,93425$, менее выраженная связь социальноэкономических условий (проживание в районах, где происходят боевые действия, перемещенные лица, неудовлетворительные жилищные условия, низкий материальный доход) $-\mathrm{r}=0,42889$ и степенью повышения АД. Не выявлено корреляционных связей с полом, возрастом больных АГ. Установлено влияние употребления ката на частоту и длительность подъема АД, средний уровень АД для данной категории больных составил 163 и 95 мм рт. ст., и это в большей степени было характерно для мужчин.

Практические рекомендации. Полученные нами данные показывают необходимость усиления лечебно-профилактических мероприятий, требуют реализации образовательного проекта как среди больных АГ, так и среди здоровых жителей Республики Йемен. Они могут также быть использованы в качестве основы для немедикаментозного лечения АГ жителей Республики Йемен.

\section{Список литературы}

1. Европейские рекомендации по профилактике сердечно-сосудистых заболеваний в клинической практике. Пересмотр, 2016. 
2. Климов А.В., Денисов Е.Н., Иванова О.В. 2018. Артериальная гипертензия и ее распространенность среди населения. Молодой ученый. 50: 86-90. URL https://moluch.ru/archive/236/54737/.

3. Чазова И.Е., Жернакова Ю.В. от имени экспертов. 2019. Клинические рекомендации. Диагностика и лечение артериальной гипертонии. Системные гипертензии. 16 (1): 6-31.

4. Al-huthi M., Jayed D., Khat Chewing Induces Cardiac Arrhythmia. Open Access. Libr. J., 2016. 3: 1-7.

5. Arnaud D. Kaze, Aletta E. Schutte, Sebhat Erqou, Andre P. Kengne, Justin B. EchouffoTcheugui, Prevalence of Hypertension in Older People in Africa: A Systematic Review and MetaAnalysis, J. Hypertens. 2017; 35 (7): 1345-1352.

6. Baker R., Camosso-Stefinovic J., Gillies C., Shaw E.J., Cheater F., Flottorp S., Robertson N., 2010. Tailored interventions to overcome identified barriers to change: Effects on professional practice and health care outcomes. Cochrane Database Syst. Rev.; 3.

7. Centers for Disease Control and Prevention (CDC). Hypertension Cascade: Hypertension Prevalence, Treatment and Control Estimates Among US Adults Aged 18 Years and Older Applying the Criteria From the American College of Cardiology and American Heart Association's 2017 Hypertension Guideline - NHANES 2013-2016 external icon.

8. Chow C.K., Teo K.K., Rangarajan S., Islam S., Gupta R., Avezum A., Bahonar A., Chifamba J., Dagenais G., Diaz R., Kazmi K., Lanas F., Wei L., Lopez-Jaramillo P., Fanghong L., Ismail N.H., Puoane T., Rosengren A., Szuba A., Temizhan A., Wielgosz A., Yusuf R., Yusufali A., McKee M., Liu L., Mony P., Yusuf S. Prevalence, awareness, treatment, and control of hypertension in rural and urban communities in high-, middle-, and low-income countries. JAMA 2013; 310 (9): 959-68.

9. Kearney P.M., Whelton M., Reynolds K., Whelton P.K., He J. Worldwide prevalence of hypertension: a systematic review. J. Hypertens. 2004; 22: 11-9.

10. El-Menyar Ayman. Qat chewing: History and heart failure. Oman. Med. J., 2015. 30: 77-82.

11. Lawes C.M., Hoorn S.V., Rodgers A. Global burden of blood-pressure-related disease, 2001. Lancet 2008; 371 (9623): 1513-8.

12. Moser M. 2006; Redon J., 2008, Resistant Hypertension: Epidemiology, Pathophysiology, Diagnosis and Treatment.

13. Nieuwlaat R., Schwalm J.D., Khatib R., Yusuf S. Why are we failing to implement effective therapies in cardiovascular disease? Eur. Heart. J. 2013; 34 (17): 1262-9.

14. Willams B., Mancia G. 2018. ESC/ESH Guidelines for the management of arterial hypertension. Eur. Heart. J. 2018. 39 (33): 3021-104, doi:10.1093/eurheartj/ehy339.

15. Who Emro High Blood Pressure A Public Health Problem World, Prevalence Of Hypertension Worldwide 2017 - URL https://prevent-hypertency.blogspot.com/2007/08/prevalence-ofhypertension-worldwide.html.

16. World Health Organization. Global health risks: Mortality and burden of disease attributable to selected major risks. Geneva: World Health Organization, 2009.

17. Ahmed Hasan. 2014. Alkhat usabeb surat darabat alkhalb wahafakanh wazyadat dagt aldam (Khat causes rapid heartbeat, palpitations and increased blood pressure) - Dar Al Akhbar Magazine Medicine - 2, 1436 date of the application 10.04.2020 - http://www.daralakhbar.com/articles/ 4619302 (in Arabic).

18. Mohtar Altaherhasan. 2005. Taber watahrer lerashedin almarhala althanawya (Expression and editing for high school) 3 (1) p. 149 (218) (in Arabic).

WHO - Khat chewing in Yemen. Bulletin of the World Health Organization Past issues Volume 2008, Volume 86, Number 10, 2008, 737-816. date of the application 10.04.2020 URL https://www.who.int/bulletin/volumes/86/10/08-011008/ar / (in Arabic).

\section{References}

1. Evropejskie rekomendacii po profilaktike serdechno-sosudistyh zabolevanij v klinicheskoj praktike [European guidelines for the prevention of cardiovascular disease in clinical practice]. Peresmotr. 2016.

2. Klimov A.V., Denisov E.N., Ivanova O.V. 2018. Arterial'naja gipertenzija i ee rasprostranennost' sredi naselenija [Hypertension and its prevalence in the population]. Molodoj uchenyj. 50: 86-90. URL https://moluch.ru/archive/236/54737/. 
3. Chazova I.Ye., Zhernakova Yu.V. ot imeni ekspertov. 2019. Klinicheskiye rekomendatsii. Diagnostika i lecheniye arterial'noy gipertonii. Sistemnyye gipertenzii (Clinical recommendations. Diagnosis and treatment of arterial hypertension. Systemic hypertension); 16 (1): 6-31. 2016. 3: 1-7.

4. Al-huthi M., Jayed D., Khat Chewing Induces Cardiac Arrhythmia. Open Access. Libr. J.,

5. Arnaud D. Kaze, Aletta E. Schutte, Sebhat Erqou, Andre P. Kengne, Justin B. EchouffoTcheugui, Prevalence of Hypertension in Older People in Africa: A Systematic Review and MetaAnalysis, J. Hypertens. 2017; 35 (7): 1345-1352.

6. Baker R., Camosso-Stefinovic J., Gillies C., Shaw E.J., Cheater F., Flottorp S., Robertson N., 2010. Tailored interventions to overcome identified barriers to change: Effects on professional practice and health care outcomes. Cochrane Database Syst. Rev.; 3.

7. Centers for Disease Control and Prevention (CDC). Hypertension Cascade: Hypertension Prevalence, Treatment and Control Estimates Among US Adults Aged 18 Years and Older Applying the Criteria From the American College of Cardiology and American Heart Association's 2017 Hypertension Guideline - NHANES 2013-2016 external icon.

8. Chow C.K., Teo K.K., Rangarajan S., Islam S., Gupta R., Avezum A., Bahonar A., Chifamba J., Dagenais G., Diaz R., Kazmi K., Lanas F., Wei L., Lopez-Jaramillo P., Fanghong L., Ismail N.H., Puoane T., Rosengren A., Szuba A., Temizhan A., Wielgosz A., Yusuf R., Yusufali A., McKee M., Liu L., Mony P., Yusuf S. Prevalence, awareness, treatment, and control of hypertension in rural and urban communities in high-, middle-, and low-income countries. JAMA 2013; 310 (9): 959-68.

9. Kearney P.M., Whelton M., Reynolds K., Whelton P.K., He J. Worldwide prevalence of hypertension: a systematic review. J. Hypertens. 2004; 22: 11-9.

10. El-Menyar Ayman. Qat chewing: History and heart failure. Oman. Med. J., 2015. 30: 77-82.

11. Lawes C.M., Hoorn S.V., Rodgers A. Global burden of blood-pressure-related disease, 2001. Lancet 2008; 371 (9623): 1513-8.

12. Moser M. 2006; Redon J., 2008, Resistant Hypertension: Epidemiology, Pathophysiology, Diagnosis and Treatment.

13. Nieuwlaat R., Schwalm J.D., Khatib R., Yusuf S. Why are we failing to implement effective therapies in cardiovascular disease? Eur. Heart. J. 2013; 34 (17): 1262-9.

14. Willams B., Mancia G. 2018. ESC/ESH Guidelines for the management of arterial hypertension. Eur. Heart. J. 2018. 39 (33): 3021-104, doi:10.1093/eurheartj/ehy339.

15. Who Emro High Blood Pressure A Public Health Problem World, Prevalence Of Hypertension Worldwide 2017 - URL https://prevent-hypertency.blogspot.com/2007/08/prevalence-ofhypertension-worldwide.html.

16. World Health Organization. Global health risks: Mortality and burden of disease attributable to selected major risks. Geneva: World Health Organization, 2009.

17. Ahmed Hasan. 2014. Alkhat usabeb surat darabat alkhalb wahafakanh wazyadat dagt aldam (Khat causes rapid heartbeat, palpitations and increased blood pressure) - Dar Al Akhbar Magazine Medicine - 2, 1436 date of the application 10.04.2020 - http://www.daralakhbar.com/articles/ 4619302 (in Arabic).

18. Mohtar Altaherhasan. 2005. Taber watahrer lerashedin almarhala althanawya (Expression and editing for high school) 3 (1) p. 149 (218) (in Arabic).

19. WHO - Khat chewing in Yemen. Bulletin of the World Health Organization Past issuesVolume - 2008, Volume 86, Number 10, 2008, 737-816. date of the application 10.04.2020 URL https://www.who.int/bulletin/volumes/86/10/08-011008/ar / (in Arabic).

\section{ИНФОРМАЦИЯ ОБ АВТОРАХ}

Вишневский Валерий Иванович, доктор медицинских наук, профессор, заведующий кафедрой внутренних болезней Орловского государственного университета имени И.С. Тургенева, г. Орел, Россия

\section{INFORMATION ABOUT THE AUTHORS}

Valery I. Vishnevsky, D. M. N., professor, head of the department of Internal Medicine, Oryol State University named after I.S. Turgenev, Orel, Russia 
Исмаил Ариф Ахмед Абдуллах, аспирант кафедры внутренних болезней Орловского государственного университета имени И.С. Тургенева, г. Орел, Россия

Аль-Гунаид Мохамед Амин, кандидат технических наук, доцент кафедры «Системы автоматизированного проектирования и поискового конструирования» (САПРиПК) Волгоградского государственного технического университета, г. Волгоград, Россия

Саид Хашем Анвар Фаид, ординатор кафедры госпитальной терапии Национального исследовательского Мордовского государственного университета имени Н.П. Огарёва, г. Саранск, Республика Мордовия, Россия
Aref Ahmed Abdullah Esmail, $\mathrm{PhD}$ student department of Internal Medicine, Oryol State University named after I.S. Turgenev, Orel, Russia

Mohammed Amin Al-Gunaid, K. T. N., associate Professor of the department of CAD and Computer Systems, Volgograd State Technical University, Volgograd, Russia

Hashem Anwar Fayd Saeed, Resident of the Department of Hospital Therapy, Mordovia State University. N.P. Ogareva, Saransk, Russia 